\title{
Germanica
}

GERMANICA $7 \mid 1990$

Grenze und Entgrenzung

\section{Mosaik, Roman in 84 Teilstücken}

\section{Irmgard Perfahl}

\section{OpenEdition}

Journals

Édition électronique

URL : http://journals.openedition.org/germanica/2517

DOI : 10.4000/germanica. 2517

ISSN : 2107-0784

\section{Éditeur}

Université de Lille

\section{Édition imprimée}

Date de publication : 30 juin 1990

Pagination : 167-187

ISSN : 0984-2632

\section{Référence électronique}

Irmgard Perfahl, « Mosaik, Roman in 84 Teilstücken », Germanica [Online], 7| 1990, Online erschienen am: 18 Juli 2014, abgerufen am 06 Oktober 2020. URL : http://journals.openedition.org/germanica/ 2517 ; DOI : https://doi.org/10.4000/germanica.2517

Ce document a été généré automatiquement le 6 octobre 2020.

(c) Tous droits réservés 


\title{
Mosaik, Roman in 84 Teilstücken
}

\author{
Irmgard Perfahl
}

\section{Teilstück 34}

1 Ist es wirklich so, dass ein Kurzsichtiger die Welt anders sieht als ein Normalsichtiger?

2 Gewiss ist es so. Ein Kurzsichtiger sieht die Dinge nicht scharf, nicht deutlich, sondern weich und verschwommen. Die Dinge wirken grösser, weil alle Ränder verschwimmen, weil Begrenzungslinien nicht als als Linien zu erkennen sind. Die kleinsten Lampen erscheinen wie grosse diffuse Feuerbälle.

Wenn ein Kurzsichtiger seine Brille aufsetzt, werden alle Dinge kleiner. Deutlicher, aber kleiner und somit doch wieder undeutlich, wenn auch auf andere Weise. Besondere Feinheiten, die ein Normalsichtiger auf weite Entfernung erkennen kann, kann ein Kurzsichtiger auf keinen Fall erkennen: ohne Brille erkennt er sie nicht, weil alles zu sehr verschwimmt und mit Brille erkennt er sie nicht, weil die Brille zu sehr verkleinert. Die diffusen Feuerbälle, die sich Edem Kurzsichtigen mit Brille als Lampen $\mathrm{zu}$ erkennen geben, erfahren also eine Verkleinerung nicht nur, weil ihre Begrenzungen sichtbar werden, sondern weil die Linsen der Brillen für Kursichtige noch zusätzlich verkleinern.

4 Der Kurzsichtige kann demnach auch mit Brille in der Ferne nicht viel unterscheiden. Dafür sind dann die Farben kräftig. Sowie er die Brille abnimmt, werden die Farben matt.

5 Der Kurzsichtige weiss nicht und wird nie wissen: sind die Farben in Wirklichkeit matt und ist es nur die Wirkung der Brille, dass sie glänzend erscheinen, oder sind die Farben in Wirklichkeit kräftig und nur die unkorrigierte Kurzsichtigkeit lässt sie matt erscheinen?

6 Vielleicht liegt die Wahrheit auch in der Mitte und ein Normalsichtiger sieht die Farben weder allzu kräftig noch allzu matt.

7 Ein Kurzsichtiger kann sich kaum vorstellen, wie ein Normalsichtiger die Welt sieht, erst recht weiss er nicht, wie ein Weitsichtiger sie sieht. Der sieht wahrscheinlich alles 
überdeutlich, er sieht wahrscheinlich die kleinsten Fehler, die einem Kurzsichtigen ganz und gar entgehen.

Ist es möglich, dass ein Kurzsichtiger gutmütiger ist als ein Weit - und sogar ein Normalsichtiger, einfach weil er die Fehler nicht sieht?

Was man nicht sieht, kann man sich schwer vorstellen. Selbst wenn man weiss, dass Fehler vorhanden sind - es machen einen ja alle Leute darauf aufmerksam - bleibt man ungläubig. Wie sollen da Fehler sein, wo man keine erkennen kann?

In seiner Kurzsichtigkeit erkennt der Kurzsichtige nur seine eigenen Fehler gut, denn sie sind ihm nahe genug. Und wenn er von sich auf andere schliesst, wie dies fast alle Menschen tun, wird er in ihnen die gleichen Fehler vermuten, die er selber hat. Ein Kurzsichtiger, der keine besonders schweren Fehler hat oder sogar nur ganz leichte, wird die Menschen also für eher gut halten und weil er sie für gut hält, ist er vertrauensvoll.

Ein Normalsichtiger (erst recht ein Weitsichtiger) hat es bedeuten schwerer, vertrauensvoll zu sein. Er sieht in den Gesichtern die Heimtücke und Bosheit, vor denen er unermüdlich auf der Hut sein muss, er sieht alle Fehler und Falschheiten.

Die groben und grossen Fehler erkennt natürlich auch ein Kurzsichtiger, ungefähr wenigstens und je nach dem Grad der Kurzsichtigkeit besser oder schlechter. Wenn er seine Brille aufsetzt, um den Fehler, den er nur ungefähr erkannt hat, besser ins Auge zu fassen, werden zwar seine Konturen deutlicher, aber auch kleiner. Der Fehler ist also schon nicht mehr so gross, wie er im ersten Augenblick, als der Kurzsichtige ihn unbewaffneten Augs bemerkt hat, erschienen ist.

Die Gutmütigkeit des Kurzsichtigen ist demnach durch einen Sehfehler bedingt. Wäre er nicht kurzsichtig, wäre er nicht gutmütig.

Weil der Kurzsichtige nichts genau sieht ausser das Allernächste, kann er auch nichts Entferntes besonders wichtig nehmen. Vor der Zukunft fürchtet er sich deshalb nicht und sein beliebtestes Sprichwort lautet: kommt Zeit, kommt Rat. Er tut sich nur in der Gegenwart um, denn die sieht er. Ob sie ihm allerdings gefallt, ist fraglich, er kann sie mit nichts Fernerem, weder in Vergangenheit noch Zukunft, recht in Verbindung bringen und so erscheint sie ihm als Inbegriff des Wirrwarrs. Mit ordnenden Händen rudert er in dem Chaos herum. Er stellt fest, dass er nichts ausrichtet, oder wenig, und kann sich daher auch selbst nicht für wichtig halten. Ausserdem wäre denkbar, dass er seine eigene Un- Wichtigkeit aus der Unwichtigkeit des undeutlichen Entfernten schliesst.

Nun könnte es sein, dass ein ängstlicher Kurzsichtiger sich just deshalb vor Entferntem fürchtet, weil das Ferne so undeutlich ist, die Zukunft beisspielsweise. Er empfindet als bedrohlich, was er nicht durchschauen kann, er verschanzt sich in sich und in seinem nahen Eigentum. Ist ein solcher kurzsichtiger noch gutmütig? Angst ist keine Förderin der Güte.

16 Andererseits - es fällt mir schwer zu glauben, dass ein Kurzsichtiger ängstlich sein kann. Gelassenheit passt viel besser zu ihm.

Wenn ich mir einen Kurzsichtigen vorstelle, sehe ich ihn am Frühstückstisch sitzen. Die Brille liegt neben der Kaffeeschale. Er liest die Zeitung, kriecht ganz in sie hinein. Kann sein, seine Frau fragt ihn etwas, dann lässt er das Blatt sinken, greift zur Brille und 
schaut seine Gefährtin an: was sagtest du? Nicht um seine Frau zu ärgern, liest er beim Frühstück die Zeitung, sondern aus Tolpatschigkeit.

Das Wort «kurzsichtig» klingt nach unsicherem Herumstolpern.

Wie klingt - bespielsweise - «myope»? Es klingt, als ginge grade vor der Nase des myope eine Schranke herunter, es klingt nach Eingeschlossensein und Darum Wissen. Elle est myope, sagt ihr Mann von ihr, elle regarde la photo de tout près, elle veut tout voir, tout reconnaître...

Alles erkennen wollen in dem engen Raum, der dem Kurzsichtigen erkennbar ist.

Ist der Kurzsichtige dumm, weil er weiter Entferntes nicht erkennen kann? Die Dummheit des Kurzsichtigen, welche Verleumdung!

Ihm bleibt nichts anderes übrig als seine Phantasie zu Hilfe zu nehmen, damit er sich ausmalen kann, was er nicht genau sieht. Wen wundert's, dass sein Phantasiegemälde mitunter merklich von der Wirklichkeit abweicht? Dass es womöglich bizarr ist, zum Kopfschütteln Anlass gibt? Soll man den Kurzsichtigen deshalb einen Kranken nennen, einen Optodeviaten? Nein, krank ist er nicht, nur sein Auge ist anders.

Was ist, wenn ein Kurzsichtiger ein Mosaik anschaut? Er kann vielleicht nicht erkennen, was es darstellen soll, besonders wenn es ziemlich entfernt hoch oben in einem eher dunklen Raum angebracht ist. Alle Figuren erscheinen ihm verschwommen, oft kann er Figuren überhaupt nicht ausmachen, er sieht bloss undeutliche Flecken. Er strengt seine Augen an. (Im Wort «myope» klingt, wenn man die Bedeutung vernachlässigt, «myo» = «Myskel» an, man spürt beim Klang von «myope» die vergebliche - Anspannung des Augenmuskels.)

Hie und da sieht der Kurzsichtige im Mosaik etwas aufblitzen, es könnten Diamanten sein, andererseits hält er Diamanten in einem Mosaik für unwahrscheinlich. Er täuscht sich wohl. Und doch ist es gerade das Gefunkel, das ihn anzieht. Alles Funkelnde wird ja riesengross für den Kurzsichtigen.

Bestimmt würde dem Kurzsichtigen das Mosaik auch ohne vermeintliche Diamanten gefallen. Er hat, auch wenn er keine oder nur wenige Einzelheiten erkennen kann, einen allgemeinen Farbeindruck von dunklem Rosa und mattem Blau und das sind gerade die Farben, die er liebt. Er bemerkt auch ein paar grünliche Flächen, die sich undeutlich im Blau verlieren. Grün liebt der Kurzsichtige weniger, aber er glaubt, im Grün stellenweise einen Goldoder Silberschimmer zu sehen und das gefällt ihm sehr.

Es kann sein, dass der Kurzsichtige im Mosaik Figuren zu erkennen glaubt, die darin nicht vorkommen. Kein Unglück. Im Gegenteil.

\section{Teilstück 35}

$\mathrm{X}$ hat im Zuge ihrer Höhlenforschung festgestellt, dass die Vergangenheiten der Menschen Höhlen sind, die Menschen kommen aus ihren Vergangenheitshöhlen gekrochen, so stellt sich's ihr dar und sie möchte nun diese Vergangenheitshöhlen untersuchen. Sie fragt alte Leute nach ihrer Vergangenheit aus, nach einer bestimmten Zeit in ihr, nach dem Krieg. Sie will Geschichte von unten betreiben. Sie hofft, alle diese Vergangenheitshöhlen zu einem Höhlensystem verbinden zu können, aus welchem etwas wie ein Geschichtsbild ablesbar sein würde. 
$\mathrm{X}$ merkt bald, dass die Leute ihre Vergangenheitshöhlen mit weichen Lügenteppichen ausgestattet haben. Der granitene Boden der Wahrheit verschwindet darrunter. Nach ihrem eigenen Zeugnis waren die Leute grösstenteils Helden, sie haben grösstenteils Übermenschliches geleistet. Einige geben zu, Angst gehabt zu haben und nur aus Angst Mitläufer gewesen zu sein, beileibe nicht aus Opportunismus. Oder sie waren Mitläufer aus Unwissenheit - wenn sie gewusst hätten.

Die Leute, mit denen X sich unterhält, sprechen aber nicht nur über sich, sondern mehr noch über die anderen. Die anderen waren grösstenteils die Schlechten, die anderen haben alles gewusst, die anderen haben denunziert, die anderen haben gemordet.

Um einen besseren Überblick zu gewinnen, sondert $\mathrm{X}$ die Icherzählungen von den Erzählungen über die anderen $a b$, sie setzt aus diesen und aus jenen je eigene Geschichtsbilder zusammen. Im Gegensatz zu dem hellen Geschichtsbild, das sich aus den IchErzählungen ergibt, fällt das Geschichtsbild, das sich aus den Erzählungen über die anderen ergibt, recht düster aus.

$31 \mathrm{X}$ trennt also die bunten Lügenteppiche aus den Vergangenheitshöhlen auf und zerlegt sie in die hellen und die dunklen Fäden. Die hellen Fäden webt sie neu, die dunklen Fäden webt sie ebenfalls neu.

Ist das dunkle Geschichtsbild aus den Taten der anderen richtiger als das helle Ich-Bild? Oder ist das dunkle Geschichtsbild ebenso falsch wie das helle, nur negativ falsch, während das helle Geschichtsbild positiv falsch ist? Würde die Methode, kongruente Stellen herauszusuchen, weiterführen? Könnte man Begebenheiten, die sowohl im dunkleren als auch im helleren Bild erscheinen, versuchsweise als wahr ansehen?

Es zeigt sich, dass keine Figur aus dem dunklen Geschichtsbild mit einer Figur aus dem hellen Geschichtsbild ganz deckungsgleich ist. Wo also ist die Wahrheit?

Nachdem X hundert Leute befragt hat, gibt sie ihre Forschungsversuche auf. Ach was, Wahrheit - für wahr hält man ohnehin nur, was man im Augenblick gerade glaubt, im nächsten Augenblick glaubt man etwas anderes.

$\mathrm{X}$ gesteht sich ein, dass sie vieles nicht berücksichtigt hat, was sehr wohl Beachtung verdient hätte. Wie sehr einer prahlt oder die anderen schlecht macht, hängt stark von seiner Erziehung und Stellung ab. Der gut Erzogene hält sich in allem zurück, der hoch Stehende hat Prahlen und Verleumden nicht nötig. Oder doch? Ist nur seine Vorgehensweise subtiler? X hätte also, um eine annähernde Stimmigkeit ihrer Geschichte von unten zu erreichen, nicht nur das dunkle und das helle Geschichtsbild übereinander legen müssen, damit sich kongruente Stellen abzeichnen könnten - sie hätte über beide noch ein durchsichtiges Korrektionsraster legen sollen, das erziehung, Stellung und Kenntnisse der Befragten berücksichtigt hätte. Zu viel Aufwand...

dass sie den Befragten ihre Lügen und Beschönigungen übelnimmt; sie ist ja auch nicht besser. Sie verschweigt, was sie falsch gemacht hat, oder, wenn dies allzu offensichtlich ist, erfindet sie tausend Gründe, um ihre Handlungsweise $\mathrm{zu}$ rechtfertigen.

Erschwerend war ferner, dass nicht alle Befragten gleich gesprächig waren. Manche kamen vom Hundertsten ins Tausendste, manche glaubten sogar, mit der Erfindung von Geschichten X eine Freude zu machen, andere dagegen baten um Verzeihung, weil sie sich nicht mehr genau oder gar nicht erinnern konnten.

Ernüchtert versteckte $\mathrm{X}$ das gesammelte Material in einer Schublade. 
Zwei Wochen später, beim Ordnungmachen, holt sie es wieder hervor, sie will es wegwerfen, bringt es aber nicht über sich. Soll die ganze Arbeit umsonst gewesen sein? Unschlüssig wägt sie die beiden Stösse gegeneinander ab, links die Ich-Aussagen, rechts die Aussagen über die anderen. Jeder Stoss ist in sich nach Ereignissen geordnet, zum Beispiel: Bombardement der Stadt K, Rückzug aus R., und so weiter. X legt die Stösse wieder hin. Sie setzt sich und denkt nach.

Sie will ihre Fragerei wieder aufnehmen, aber nun wird sie nach Zuständen fragen und nicht nach Ereignissen, zumindest nicht nach geschichtlichen Ereignissen, sondern nach privaten: Wie hat die Stadt ausgesehen, als Sie ein King waren, wie war das damals in der Schule? Die Gesichter der Befragten öffnen sich, Lächeln erscheint langsam auf ihnen, in den Augen spiegelt sich längst vergangenes Glück... In der Schule, mein Gott ja, da hat der Schuldiener im Hof mit einer grossen Handglocke die Stunden und die Pausen eingeläutet... Ans Schulhaus grenzten damals Felder und ein kleines Wäldchen, das war fein für uns Kinder, wir haben dort Verstecken gespielt, jetzt stehen da lauter Hochhäuser...

Und als ihr jung wart, wie war's als ihr jung wart?... Wir haben im Fluss gebadet, der Fluss war noch klar. Und hinter den Büschen - dort haben wir uns ausund angekleidet haben wir die ersten Küsse getauscht, in der Nacht, wenn wir dran dachten, hat uns das Herz geklopft...

Es öffnen sich schöne freundliche Vergangenheitshöhlen, die nicht mit Rechtfertigungsund Verleumdungsteppichen ausgelegt sind, sondern mit Erinnerungsflickenteppichen, mit bunten, stellenweise etwas abgechabten, vielleicht auch nicht farbechten, nicht wahrheitsentsprechenden Geweben - was tut's, sie machen die Vergangenheitshöhlen behaglich.

Wir haben getanzt, getanzt haben wir, ganze Nächte lang haben wir getanzt. Langsame Walzer haben wir getanzt und uns in die Augen geschaut, ich tanze mit dir in den Himmel hinein, in den siebenten Himmel der Liebe. ... und Tango haben wir getanzt, ach Tango, immer wieder Tango...

Tango?

Tango, ich berühre.

$\mathrm{X}$ durchwandert die fremden Vergangenheitshöhlen, ihre Seele berührt vergangenes Glück und Sehnsüchte, sogar verklärtes vergangenes Unglück, die in den Vergangenheitshöhlen zur Ruhe gekommen sind. Die Höhlen füllen sich mit magischem blauen Licht, es ist ein dunkelblaues, trotzdem durchsichtiges Licht.

$[\ldots]$

\section{Teilstück 36}

Ich sehne mich nach einem Stückchen Paradies.

Ich sehne mich nach einem Dolce far niente, das ich nicht meinem Reichtum verdanke.

Ich möchte es meiner Bedürfnislosigkeit verdanken.

Ich mache die Augen zu.

Träume ich? Vielleicht träume ich. 

fällt, es ist Goldstaub. Von einem unendlich hohen Felsen fallt der Goldstaub an mir vorbei in einen See zu meinen Füssen, der aussieht wie dunkles Gold, doch von allerleichtester Flüssigkeit. Manchmal bläst eine blütenduftende Brise in den Goldfall, so dass Goldwolken davonstieben. Es ist kein Rauschen oder Gedonner, das der Goldfall verursacht, es ist ein zartes Klingeln, eine betörende Musik. Ich werde nicht müde, ihr $\mathrm{zu}$ lauschen - tagelang, wochenlang, jahrelang lausche ich ihr, jahrzehntelang immer?

Immer? Ich weiss nicht. Eines Tages entschliesse ich mich, hinunterzusteigen und in dem goldenen See zu baden. Ist es der See des Vergessens? Ich entsteige ihm wieder, vergoldet, aber ich habe vergessen, wer ich bin, ja, ich habe vergessen, dass ich überhaupt ein Ich bin. Augenblick, aber der Augenblick ist immer. Das Wesen ist in die Seligkeit eingegangen. Hört es noch das Goldstaubgeklingel? Das Wesen weiss es nicht. Es hat einen himmlischen Ton im Ohr, der ihm wohltut. Sieht es den Goldfall? Das Wesen weiss es nicht. Es hat etwas Leuchtendes vor dem Auge, das ihm wohltut. Riecht es noch die Blütendüfte? Es hat einen wohltuenden Duft in der Nase, auf dem Gaumen schmeckt es etwas wie Nektar, wohltuend. Auf seiner ganzen Haut spürt das Wesen eine wohltuende Berührung. einziges schreckliches Wort macht sie schon rückgängig, ein Donner von einem Wort: wach auf! solche grausamen Menschen gibt es; gibt es übergenug und manche meinen es nicht einmal schlecht. Aber von ihnen will ich jetzt nicht reden. Ich habe die Augen noch zu. Träume ich? Vielleicht traüme ich.

Ich sehne mich nach einem Dolce far niente, das ich meiner Bedürfnislosigkeit verdanke. Ich möchte es nicht meinem Reichtum verdanken. Ich sehne mich so sehr nach einem Stückchen Paradies.

\section{Teilstück 37}

Das hat man früher gesagt, wenn man von etwas Zukünftigem sprach. Man sagt es noch heute und meint damit eine ungewisse Zukunft. Es steht zwar in den Sternen geschrieben, soll das heissen, aber in einer für uns nicht lesbaren Schrift. Freilich, es hat immer Sterndeuter gegeben, die behauptet haben, die Sternenschrift entziffern zu können und auch heute gibt es übergenug davon. Verwegene Astrologen!

Wer das für sie Herauslesbare in die Sterne geschrieben hat, erörtern sie nicht weiter, es könnte Gott gewesen sein, oder das gleichgültige Schicksal oder gar die parteiische Vorsehung. 

zu halten, wenn die beiden auseinanderdriftenden Entwurfslinien zueinander in einem spitzen Winkel stehen. Was aber, wenn die göttliche und die menschliche Entwurfslinie in entgegengesetzten Richtungen auseinanderstreben? In diesem Fall dürfte die konkrete Zukunft gänzlich überfordert sein, von Durchwursteln wird man nicht mehr reden können. Eine Resultante zwischen den beiden Entwurfslinien ist nicht möglich. Eine konkrete Zukunft, Gegenwart werdende Zukunft, gibt es dann nicht. Und wenn es keine konkrete Zukunft gibt, gibt es gar keine Zukunft. Was soll das aber heissen, gar keine Zukunft?

keine Zukunft kann für uns Menschen nur heissen, dass die Menschen verschwinden. Dies bedenkend, wird Gott sich möglicherweise aus Gutmütigkeit zurückziehen - und nicht aus Groll, wie oben angedeutet. Er gönnt es den Menschen, dass sie ihrer eigenen Entwurfslinie folgen, er verzichtet auf die Sternenschreiberei wie auch das Schicksal, die Vorsehung verzichten. Ungehemmt dürfen dann die 
Menschen in die Zukunft rasen. Sie werden immer schneller oder sagen wir lieber, die Zukunft wird schneller. Wenn sie eines Tages Lichtgeschwindigkeit erreicht, was dann?

Nun komme ich auf die Frage zurück, wohin Gott - das Schicksal - sich zurückziehen könnten, wenn sie das wollten: ins Nichtsein? Als ob das so einfach wäre.

Aber vielleicht ist es für Gott sogar einfach, weiss mans? Das Schicksal und die Vorsehung lasse ich jetzt unter den Tisch fallen, für die will ich mir nicht den Kopf zerbrechen. Gott wird sich im Nichtsein vielleicht wohlfühlen. Prekär wird die Sache nur, wenn Gott womöglich von Anfang an im Nichtsein war, denn wohin könnte er sich in diesem Fall zurückziehen? Aber was rede ich! Ich habe doch an der Existenz Gottes nie gezweifelt!

Also noch einmal: Gott existiert, aber weil er uns Menschen nicht hindern will, in unsere eigene Zukunft $\mathrm{zu}$ rasen und darin eventuell unterzugehen, zieht er sich freundlich ins Nichtsein zurück, er könnte aus dem Nichts, aus sich selbst, wieder sich selbst und auch eine neue Welt erschaffen, die keine Ähnlichkeit mit unserer jetzigen hat. Die kann er dann betreuen, ihre Zukunft in die Sterne schreiben. Mag inzwischen die unsrige in ihr Unglück oder wohin auch immer torkeln.

Spielt beim Rückzug Gottes ins Nichts nicht doch ein bisschen Groll mit? Jedenfalls wird ER auf die frische junge neue Welt besser aufpassen müssen, von Anfang an, er sollte nicht so leichtsinnig sein, sich ein Ebenbild zu schaffen.

Keine Menschen, keine Sprache.

Keine Sprache, keine Blasphemien.

\section{Teilstück 38}

rücksichtsvoller, ein sanfteres Lecken im Ohr, wohltuend, ein zarte Schmeichelei. La langue ist doppeldeutig, heisst Zunge und Sprache und in jeder seiner Bedeutungen schwingt unterschwellig etwas von der anderen Bedeutung mit. La langue, die Zunge, ganz nah und intim und zärtlich und doch die Möglichkeit sprachlicher Verständigung in sich enthaltend. La langue, die Sprache, fern und rational und diplomatisch und doch die Möglichkeit inniger Liebkosung, die Möglichkeit des Schmeckens enthaltend.

81 Bei uns fällt's auseinander. Hier die Zunge, die schmeckt und leckt, dort die Sprache, ein Instrument des Geistes, im Klang nüchtern. Im Klang so trocken wie aufgereihte Kugeln auf einem Rechenbrett, die man von einer Seite auf die andere schubst. Noch ähnlicher wäre das Kugelgeräusch dem Wort Sprache, wenn man aus ihm die Vokale wegliesse: SPRCH, sind alle Kugeln auf der anderen Seite, SPRCH sind sie wieder zurück mit hölzernem Geräusch.

Germanica, 7| 1990 
Schriebe man allein die Konsonanten, könnte man nicht mehr wissen, soll es SPRACH heissen oder SPRACHE, SPRAECHE, SPRECHE, SPRICH, SPRUCH oder SPRUECHE. Von einem, der seine Sprache aufplustert, sagt man, er macht Sprüche. Ist der Anfang der Vokalreihe etwa hochwertiger als ihr Ende, gibt es einen Abfall von A über die übrigen Vokale zu U, Beweis: die Wahrheit, aber Lug und Trug? Ist Sprache um vieles besser als Sprüche? Die Sprüche, angesiedelt am dunkelsten untersten Ende der Vokalskala, haben mit der Sprache wirklich wenig gemein.

Mehr hat Sprache mit langue zu tun, beide Worte enthalten dieselben Vokale. Nur lässt sich in das Wort langue die Zunge viel leichter hineingleiten als in das Wort Sprache. Bei diesem ist ein Konsonantenhindernis, einKonsonantengestrüpp, ein Konsonantendrahtverhau zu überwinden, bevor man den Vokal A erreicht. Die deutsche Zunge, gewöhnt an derlei Drahtverhaue, überwindet es bravourös. SPR ... Will die deutsche Sprache, indem sie solche Hindernisse aufbaut - und gerade in dem Wort Sprache - damit andeuten, dass es schwer ist, in die Sprache zu kommen, zum Sprechen zu kommen? Wenn man einmal drin ist im Wort Sprache, im langen A sich badet, ist alles gut. Hinaus kommt man leicht. Ein leichtes Kratzen im Hals, ein kurzes zartes Gekrächz, CH ... schon ist man im E, das rasch verhaucht wird.

Haben wir das Wort Zunge als Synonym für Sprache aufgegeben, weil es uns zu wenig daraufhinwies, wie schwierig es ist, zur Sprache, zum Sprechen zu kommen? Ach was, der Nichtgebrauch von ZUNGE für SPRACHE wird andere Gründe haben. Es wird wohl reinste Prüderie sein, nicht von ZUNGE zu reden, wenn man SPRACHE meint, die tief ins Ohr dringen soll.

\section{(...} ..)

Die Zukunft, l'avenir.

Das Zukünftige, le futur.

Die Zukunft und l'avenir habe ich schon einmal gegeneinandergestellt, die Zukunft als das Düstere, Verschlossene, l'avenir als das Helle, offene. Das Zukünftige, der Inhalt der Zukunft, das was sie uns bringen will, sieht in beiden Sprachen schon ähnlicher aus. Das Zukünftige, le futur, düster, wenn nicht fürchterlich.

Das düstere Zukünftige ist unserer dunklen Zukunft gemäss. Wie steht es aber mit dem düsteren futur in dem hellen avenir? Est-ce possible que l'avenir clair soit capable de dissoudre le futur obscur? Espérons-le.

(...)

\section{Teilstück 42}

$\mathrm{U}$ ist nicht mit allem zufrieden, was $\mathrm{X}$ macht. Ihre Fragerei mittels welcher sie Geschichte von unten betreiben will, mißfallt ihm gründlich. Alles Fragen ist Eindringen.

«Oh» sagt X, «und?»

«Willst du gefragt werden?»

«Warum nicht? es hängt davon ab, wer mich fragt, und was. Wenn mir eine Frage nicht paßt, gebe ich keine Antwort».

«Fragen sind auf Antworten bedacht, solche, auf die keine Anwort erfolgt, sind wie in die Luft verschossene Pfeile. Aber, liebe X, glaubst du, es ist so einfach, nicht zu antworten? Wenn 
man dich fragt, wirst du auf jeden Fall irgend eine Antwort geben. Auch keine Antwort ist eine Antwort, sagt man».

«Nicht a \if eine bestimmte Frage. Ich sage dann, darauf antworte ich nicht».

Ein Fragender könnte aber damit nicht zufrieden sein und weitere Fragen stellen.

«Laß ihn nur, laß ihn», sagt X, «man kann ja falsche Antworten geben, auf jeden Fall kann man sich Fragen leicht entziehen».

«Leicht? Fragen halten den Befragten fest. Denn die Wirkung der Fragen auf den Fragenden ist eine Hebung seines Machtgefühls».

Und Xens Replik: Klug ist eine Antwort, die dem Fragen ein Ende macht.

«Siehst du», triumphiert U, «Du hast dich schon ganz in den Befragten hineinversetzt. Du wirst nicht mehr fragen. Und ich sage dir noch etwas: Die Frage, die letzten Endes auf Zerlegung aus ist, beginnt mit Berührung».

Die Frage, die letzten Endes auf Zerlegung aus ist, beginnt mit Berührung? fragt X entsetzt. Sie fühlt sich brutal herumgerissen, plötzlich mit einem Sinn konfrontiert, der vorher nicht zugegen war. Tango, denkt sie und greift mit den Händen in die Luft. Berührung. Ich berühre. Tango ganze Nächte haben wir getanzt, $\mathrm{X}$ ist schwindlig, alles dreht sich um sie, sie hat die weiche Stimme einer Befragten im Ohr, Tango.

«Was ist?» fragt U besorgt.

«Nichts».

«Mach mir nichts vor. Was ist?»

«War meine Fragerei auf Zerlegung aus? Nicht vielmehr auf Zusammensetzung? Zusammensetzung eines Geschichtsbildes? War denn Berührung der Anfang der Fragerei, nicht vielmehr ihr Ende? Ich habe mich so sehr gefreut, als ich bei den Erzählungen über Walzer und Tango auf etwas Echtes gestoßen bin - geglaubt habe, gestoßen zu sein - hier, als ich Privates berührte, so schien es mir, war endlich die Berührung mit der Wahrheit. Vorher hatten mir die Leute allerlei Ausfchneidereien, Übertriebenes, Falsches, Verlogenes aufgetischt. Als ich nicht mehr fern und inquisitorisch gefragt habe, was haben Sie damals gemacht, sondern nah und freundlich, wie war's denn damals, habe ich erschöpfende Antworten bekommen».

«Und du glaubst, daß diese Antworten keine Aufshneidereien waren?»

«Vielleicht Auschneidereien, aber nicht falsch. Den inquisitorischen Fragen haben sich die meisten Leute verweigert, sie haben gar keine oder unrichtige Anworten gegeben, sie sind nicht in sich gegangen, niemand hat eine Schuld bekannt. Klug ist eine Antwort, die dem Fragen ein Ende macht. Die Leute haben durch ihre falschen Antworten dem Fragen ein Ende gemacht, ich habe diese Art zu fragen aufgegeben. Dazu kommt, daß ich keine Amtsperson bin und eigentlich zum Fragen keine Berechtigung, keine Ermächtigung hatte. Ich frage mich, ob ich als Amtsperson redlichere Antworten bekommen hätte. Man muß eine gewisse Machtstellung schon haben, um einzuschüchtern; dann steigert sich die Sache von selbst: Denn die Wirkung der Fragen auf den Fragenden ist eine Hebung seines Machtgefühls...».

«Auch eine Amtsperson», sagt U, «könnte die falschen Fragen stellen, so daß das Ergebnis kümmerlich ist und das Machtgefühl sich nicht nennenswert hebt. Sie erhält veilleicht Antworten, die ihrerseits Fragen sind. Ein Fragender könnte aber damit nicht 
zufrieden sein und weitere Fragen stellen. Solche Fragen können immer peinlicher werden, sie können sich zum Verhör und das Verhör zur Tortur».

«Ich war doch weit davon entfernt, irgend eine Tortur anzuwenden, auch keine verbale, von Verhör konnte keine Rede sein. Und peinlich wurden die Fragen höchstens für den, der ein Gewissen hat; der wand sich vielleicht unter ihnen. Die Anderen wurden damit leicht fertig, ihre Antworten waren so gut wie keine Antworten. So war's ja wirklich: Fragen sind auf Antworten bedacht, solche, auf die keine Antwort erfolgt, sind wie in die Luft verschossene Pfeile. Ja so war's, aber das habe ich zu spät erkannt. Und in die Luft verschossenen Pfeilen - an letztlich ins Leere gerichteten Fragen konnte mir nichts gelegen sein, ich wollte eindringen, ich wollte in die Vergangenheitshöhlen eindringen und sie durchforschen. Alles Fragen ist Eindringen». «eindringen kann ein Akt der Persönlichkeitsverletzung sein». «Kann, ja. Aber: Alles Eindringen beginnt mit Berühren. Gibt es nicht auch zarte Berührung? Voll Rücksicht? Eindringen als Akt der Liebe».

\section{aus Teilstück 55}

«Die Sprache ist ein Virus aus dem fernen All.» (W.S. Borroughs)

$\mathrm{R}$ möchte eine Diagnose stellen. Er will die Ausprägungen oder Erscheinungsformen der Krankheit Sprache im Deutschen untersuchen, er sucht und findet eine Buchstabenverbindung, die er für besonders deutsch hält, für urdeutsch, es ist dies die Konsonantenverbindung $\mathrm{KN}$ am Vortanfang.

Es knackt.

Es knallt.

Der Knabe knallt mit der Knarre.

Der Knabe als Knacki im Knast.

Dies ist Neudeutsch.

Neudeutsch ist Urdeutsch hier.

Knecht und knechtisch sind etwas in Vergessenheit geraten. Knecht will niemand mehr sein. Trotzdem sollte man die Worte wieder häufiger gebrauchen. Warum «servil» sagen, wenn es das Wort «knechtisch» gibt? Die Deutschen sind servil, hört man oft, niemand sagt, die Deutschen sind knechtisch. Ja, warum wohl? Servil deutet immer noch eine Art Dienstleistung an, etwas nicht Ehrenrühriges, ja sogar die Schlauheit des Sichumstellens, gesunden Opportunismus. Knechtisch ist bloss tölpelhaft unterwürfig. Der isst Knoblauch, der Knecht, Knödel und Kartoffelknollen. Eine Knollennase hat, Knopfaugen, dumme. Verkniffen schaut er drein, aber Kniffe kennt er keine.

$\mathrm{R}$ sieht, dass es schwierig sein dürfte, die Worte Knecht und knechtisch wieder in der Umgangssprache zu verankern. Denn Kniffe kennt heute fast jeder und ist stolz darauf, allerdings nennt jeder die Kniffe lieber Tricks, um von diesem urdeutschen KN abzulenken, wegzukommen. Ohne Tricks ist das Leben heute nicht zu meistern und ein reiner Tor will niemand sein.

Dem Wort Knabe haftet freilich etwas Rein-Törichtes oder aber etwas Musterhaftes an. 
Musterhaft kann der mit der Knarre knallende Knabe allerdings nur sein als Soldat. Oder als Polizist. Als törichter findet er sich wieder als Knacki im Knast.

Dort wird ihm das Tumbe schnell ausgetrieben, dort knobelt er allerlei aus, dort rollt er für seine Kassiber Garnknöuel aus, knüpft Verbindungen an, macht sich Knoten ins Taschentuch, damit er nichts vergisst, die Rache nicht vergisst. Er knurrt vor Wut, knirscht mit den Zähnen, wenn er die knarrenden Stiefel des Wachhabenden hört, er knallt sich aufs knarzende Bett, (und er knotzt sich auf dem Bett, würde man in Osterreich sagen), er knurrt vor Enttäuschung, so knapp ist der, den der Knabe erschiessen wollte, dem Tode entronnen, so knapp! Der wird sich rächen wollen, der Entronnene, aber, die Rache ist mein, spricht der Knacki, knie nieder, du Kniich, zum Gnick-schuss!

$\mathrm{KN} .$.

Kniet nieder, ihr Knilche, zum G'nickschuss, gleich knattern die Salven! KN.... Urdeutsch.

Mitnichten. Salven und G'nickschuss, das gibt's auch woanders.

Urdeutsch etwa doch? Die Knorrigen. Gleich knattern die Salven.

Die Knorrigen: verknorpelt ihre Ethik, verhärtet, hart wie Knuppstahl. Knüppeln alles nieder, die Knorrigen. Alles knickt in die Knie, jammert um Knade.

Die knorrigen Helden landen den grossen Knüller.

$\mathrm{R}$ ist unglücklich...

Es muss doch möglich sein, KN ... auch anders zu verknoten ein Knäuel aus Goldfaden aufzurollen, zartere Netze zu knüpfen.

Das süsse kleine Mädchen knickt im Knie zum Knicks, ihr Kleidchen aus Taft knistert ein bisschen, knittert ein bisschen.

Des Knaben Sonntagshose hat den perfekten Kniff, die Knöpfe der Jacke sind goldfarben.

7 O Kinder, nur kein Lampenfieber, zerknüllt euer Taschentuch nicht. Kneifen gibts nicht, sagt eure Gedichte schön auf, zur Belohnung gibt's knusprige Brezeln zum Knabbern und Knallbonbons.

Der Knabe beginnt: «Auf den Knauf seines Schwertes gestützt blickt der Knappe...».

Dann das Mädchen: «Ein Knusperhäuschen stand im Wald...».

Knusperhäuschen? Hexenhaus! Im Knusperhäuschen wohnt die böse Hexe.

Und Schwertknauf? Ein anderes Gedicht, lieber Knabe, denk nicht immer an Schlachten.

Und der Knabe - kein Gedicht, sondern Prosa: «Die knochenharten Gangster knebeln die Geiseln...»

Schluss! Sonst kommt er noch mit der Knute, die die Geknebelten bearbeitet...

Nicht schon wieder! KN... soll nicht schon wieder die Richtung zu Tod und Verderben einschlagen. Gibt es denn ausser Knicks und Knittern kein KN-Wort, das nicht zum Verderben hin schaut?

Sogar das harmlose Knistern lässt an Vernichtung denken: das Feuer knistert unheilvoll, bevor es braust und alles verbrennt. 
Ach ja, kneten! Man knetet den Teig, bäckt knuspriges Brot, aber sogar die gute Tätigkeit des Knetens hat etwas Gewaltsames.

Was soll man machen? KN... hat eine fatale Neigung, in die Nähe von Tod und Vernichtung zu rücken. Der Pillen-Knick: Plötzlich noch mehr menschliche Keime, die zum Leben nicht zugelassen werden. R glaubt zwar, dass der Pillenknick eine optische Täuschung ist. Ein Glasstab, der schief in einem mit Wasser gefüllten Glas steht, erscheint durch die Wasseroberfläche geknickt. So erscheint auch die seit langem geradlinig nach unten verlaufende Kurve der Geburtenrate dort geknickt, wo sie ins Pillenzeitalter eintaucht. Aber wie dem auch sei, das Wort Knick suggeriert Tod.

Man knickt einen Stengel, eine Blume.

\section{Teilstück 78}

Was wissen wir denn schon, sagt $\mathrm{R}$ oft. Wir behaupten, dies stimme und jenes stimme nicht und im nächsten Augenblick sehen wir es umgekehrt und im übernächsten Augenblick zweifeln wir auch daran. Immerhin ist leichter festzustellen, was nicht stimmt als was stimmt, Gewißheit gibt es nicht, Mutmaßung ist alles.

Aber nein, Mutmaßung ist nicht alles. Der Vokal A ist keine Mutmaßung, er ist eine Gewißheit. Es gibt ihn! Nebst allen anderen Vokalen und Konsonanten. Die Vokale und Konsonanten sind Gewißheiten, aber wie das Alphabet entstanden ist, ist eine Mutmaßung. Warum das a am Anfang? War es womöglich der erste Laut, den Menschen hervorbrachten, als sie sprechen lernten? Hat man sich gefragt, was würde denn am besten zum A passen? Schon seltsam, daß man auf diese - oder eine andere - Weise auf's B gekommen ist und dan auf's $C$.

Vielleicht stößt man auf eine Gesetzmäßigkeit, wenn man die Vokalverteilung anschaut. Zwischen $\mathrm{A}$ und $\mathrm{E}$ gibt es drei Konsonanten, zwischen $\mathrm{E}$ und I ebenfalls. Zwischen I und $O$ fünf, zwischen $O$ und $U$ ebenfalls fünf. Vom U zum A wären wieder fünf wenn man $Y$ als Konsonanten zählt, was ein Schwindel ist. Aber bitte. Irgend etwas muß man immer unter den Tisch fallen lassen, wenn man zu Gesetzen kommen will. Zwei mal je drei Konsonanten zwischen den Vokalen, drei mal je fünf. Wenn das Alphabet weiterginge, kämen jetzt fünf mal je acht Konsonanten, danach acht mal je dreizehn, und so weiter, die Dibonnaci-Folge. So viele Laute kann man sich freilich nicht vorstellen. Würde man das Alphabet vom A aus ins Minus verlängern, wäre da zunächst ein einmaliger Zwischenraum von zwei Konsonaten. Es wäre das aber kein echtes Minus. Es wäre einfach der echte Anfang des Alphabets. Unser Alphabet, kann man feststellen, fangt eben nicht am Anfang der Fibonacci-Reihe an.

2 So ist es mit allen Geschichten. Wenn wir anfangen $z u$ erzählen, haben sie in Wirklichkeit schon lange angefangen, aber wann, wissen wir nicht. Die Geschichten haben ihren Ezrzählanfang lange nach ihrem wirklichen, aber natürlich nur gemutmaßt wirklichen Anfang und sie haben ihr Erzählende lange vor dem wirklichen, aber natürlich nur gemutmaßt wirklichen Ende. Vorausgesetzt, daß es Anfang und Ende überhaupt gibt.

3 Ist also das Alphabet schlicht ein Symbol für Erzählbarkeit? Was würden dann die verschiedenen konsonantischen Zwischenstrecken bedeuten? Und die gesetzmäßig verteilten Vokale? Vielleicht bedeuten die Vokale eine Aufhellung und ein kurzes ZurRuhe-kommen dramatischer konsonantischer Geschehnisse. Dann könnte man 
schließen, daß die Aufhellungen anfangs dicht aufeinander folgen, später immer weniger und weniger dicht. Zum Schluß wäre, wenn man sich das Alphabet ins Unendliche verlängert vorstellt, sozusagen kein Vokal mehr da, die konsonantischen Zwischenräume sind dann einfach schon zu groß. Daraus wieder könnte man schließen, daß jede Geschichte in konsonantischem Dunkel endet oder sich verliert. Die dramatischen tragischen Ereignisse werden von keiner Aufhellung mehr unterbrochen. Alle Geschichten enden schlecht, wenn man sie lange genug weitererzählt. Wahrscheinlieh endet unsere Menschengeschichte schlecht, wenn wir sie lange genug weiterbetreiben.

Aber das Alphabet, so wie wir es kennen, macht uns die Freude, nach dem U mit einer nicht zu langen konsonantischen Zwischenstrecke wieder auf das A zuzusteuern. Könnte dieser Sachverhalt ein Symbol dafür sein, daß alle Geschichten Wiederbeginnen, ja, daß es eigentlich immer wieder dieselben Geschichten sind? Oder ein Symbol dafür, daß wir uns weigern, das letztendliche zwangsläufige Scheitern unserer Menschheitsgeschichte ins Auge zu fassen?

Erinnern wir uns, daß die Laute an sich Gewißheiten sind, ihre Zusammens tellung aber jederzeit geändert werden könnte. Allerdings ändern wir sie nicht. Könnte dieser Sachverhalt ein Symbol dafür sein, daß wir uns auch in den Geschichten, die wir erzählen, an eine bestimmte Reihenfolge halten, obwohl sie nicht zwingend ist? Oh nein, eher umgekehrt. Das Leben hält eine bestimmte Reihenfolge ein und das Alphabet sucht diese Unabänderlichkeit auszudrücken, indem es immer gleich bleibt. Wie dem auch sei, der Vorschlag, das Alphabet zu verändern, stieße jedenfalls auf Ablehnung.

Wir können uns vorstellen, aus dem Alphabet, aus den gegebenen Lautbausteinen, völlig neue Worte zu bilden. Aber wir tun es nicht. Welche Scheu hält uns davon ab? Oder ist es Unfähigkeit? Unsere Phantasie betätigt sich hauptsächlich mit der Abkürzung und der Zusammensetzung von Worten, die wir schon haben, auch mit der Hinzuziehung und Veränderung von Wörtern aus fremden Sprachen, und mit der Abschleifung zu Umgangssprachlichem.

157 Soll man in den Kulturministerien Worterfinder anstellen, wie man in Fabriken Konstrukteure, Erfinder von Maschinen und Werkzeugen anstellt? Wozu? Noch haben wir kein Bedürfnis nach gänzlich neuen Bezeichnungen. Es könnte aber sein, daß die Bevölkerungsexplosion eines Tages von der Sachenexplosion übertroffen wird, dann vielleicht, dann wird es so viele neue Sachen geben, daß die bisherigen Worte zu ihrer Bezeichnung nicht ausreichen. Dann wird der Tag der Worterfinder gekommen sein. Könnten wir ihnen nicht schon ein bißchen vorarbeiten? Daß sie dann nicht überfordert sind.

Und was, wenn die Sachenexplosion ausbleibt? Dann stehen wir mit unseren Worterfindungen da und sehen keine Möglichkeit zu ihrer Anwendung. Ließen sich aus ihnen vielleicht hübsche Lautgedichte machen? Warum nicht? Wenn man sie auch nicht versteht wird man sicher etwas empfinden beim Lesen oder Anhören.

159 Ach, empfinden und verstehen! Diese uralten Gegensätze. Sind sie denn Gegensätze? Wenn ich bei so einem Lautgedicht irgend etwas empfinde, dann versteh ich es doch eigentlich? Wenn mir jemand schuschuschuschuschuschuschuschusch ins Ohr raunt, werd ich das doch nicht als Beleidigung empfinden? Ich könnte allerdings glauben, er wolle mich beruhigen, wo ich doch gar nicht beruhigt werden muß,da ich ja ruhig bin und könnte mich deshal büber ihn ärgern. Ich könnte aber auch in sein schuschusch einstimmen, bis wir beide über unser sinnloses schuschuschusch lachen müßten. Ich 
könnte ganz geduldig zuhören und denken, es klingt, als schu, als schu, als hischten Nachtgeister durch dunkle Wälder.

Ich schwanke ja immer, ob ich meine angeborene Empfindsamkeit vertiefen oder ob ich mich zur Gelassenheit erziehen soll. Gelassenheit gegenüber Gedichten? Hat man dann was von ihnen? Kaum. Also selektive Gelassenheit. Wenn mich einer verachtet, beleidigt, mir böse mitspielt: bleib ruhig mein Herz.

161 Aber bei Gedichten, Worten, Silben, Lauten von weher Schönhei, von schneidender diamantener Schärfe, von weiche zerschmelzende Sanftheit, da bleibe nicht ruhig, mein Herz. Denn in diesen Gedichten, Worten, Silben, Lauten, ob du sie kennst oder nicht kennst, ist die Sehnsucht der Menschen eingegangen.

Verstehst du das?

163 Alle Geschichten, die man erzählt, in jedem Alphabet, in jeder Sprache, handeln von der Sehnsucht und die Sehnsucht geht nach der Liebe und geht nach dem Unbekannten. 